\title{
Substrate arrays of iridium oxide microelectrodes for in vitro neuronal interfacing
}

\section{Shady Gawad 1,2, Michele Giugliano ${ }^{3,4}{ }^{*}$, Marc Heuschkel5, Börge Wessling $^{6}$, Henry Markram ${ }^{3}$, Uwe Schnakenberg ${ }^{6}$, Philippe Renaud ${ }^{1}$ and Hywel Morgan ${ }^{2}$}

\author{
1 Laboratory of Microsystems, Ecole Polytechnique Fédérale de Lausanne, Lausanne, Switzerland \\ 2 School of Electronics and Computer Science, University of Southampton, Southampton, UK \\ ${ }^{3}$ Laboratory of Neural Microcircuitry, Brain Mind Institute, Ecole Polytechnique Fédérale de Lausanne, Lausanne, Switzerland \\ ${ }^{4}$ Department of Biomedical Sciences, University of Antwerp, Wilrijk, Belgium \\ ${ }^{5}$ Ayanda Biosystems SA, Parc Scientifique, Ecole Polytechnique Fédérale de Lausanne, Lausanne, Switzerland \\ ${ }^{6}$ Institute for Materials in Electrical Engineering I, RWTH Aachen University, Aachen, Germany
}

\section{Edited by:}

Sergio Martinoia, University of Genova, Italy

\section{Reviewed by:}

Alexandre Schmid, Ecole Polytechnique

Fédérale de Lausanne, Switzerland

Thomas Phely-Bobin, Materials

Technology Foster-Miller, Inc., USA

${ }^{*}$ Correspondence:

Michele Giugliano, Department of Biomedical Sciences, University of Antwerp, Universiteitsplein 1, B-2610 Wilrijk, Belgium.

e-mail:michele.giugliano@ua.ac.be
The design of novel bidirectional interfaces for in vivo and in vitro nervous systems is an important step towards future functional neuroprosthetics. Small electrodes, structures and devices are necessary to achieve high-resolution and target-selectivity during stimulation and recording of neuronal networks, while significant charge transfer and large signal-to-noise ratio are required for accurate time resolution. In addition, the physical properties of the interface should remain stable across time, especially when chronic in vivo applications or in vitro long-term studies are considered, unless a procedure to actively compensate for degradation is provided. In this short report, we describe the use and fabrication of arrays of 120 planar microelectrodes (MEAs) of sputtered Iridium Oxide (IrOx). The effective surface area of individual microelectrodes is significantly increased using electrochemical activation, a procedure that may also be employed to restore the properties of the electrodes as required. The electrode activation results in a very low interface impedance, especially in the lower frequency domain, which was characterized by impedance spectroscopy. The increase in the roughness of the microelectrodes surface was imaged using digital holographic microscopy and electron microscopy. Aging of the activated electrodes was also investigated, comparing storage in saline with storage in air. Demonstration of concept was achieved by recording multiple single-unit spike activity in acute brain slice preparations of rat neocortex. Data suggests that extracellular recording of action potentials can be achieved with planar IrOx MEAs with good signal-to-noise ratios.

Keywords: substrate microelectrodes, extracellular recordings, electrophysiology, impedance spectroscopy, neurons, cortex

\section{INTRODUCTION}

A number of reports, documenting the use of IrOx microelectrodes for neuroengineering and biomedical applications, have been recently presented in the literature. The interest in this material is driven by its excellent properties as a functional coating for implantable stimulation electrodes, with applications in stimulating/recording heart, neuronal or retinal tissues (Blau et al., 1997; Cogan, 2006; Marzouk et al., 1998; Mokwa, 2007). High-resolution electrical stimulation and recording directly benefits from miniaturization of the electrodes, whilst retaining a high and reversible charge capture and delivery capacity (Cogan et al., 2005; Wessling et al., 2006). Although electrode coating with nanoparticles demonstrates excellent performances both in stimulation (Mazzatenta et al., 2007) and recording (Gabay et al., 2007; Gabriel et al., 2008; Keefer et al., 2008), the regenerative electrical activation of IrOx electrodes is simpler and faster than nanotechnological fabrication or deposition processes.

In applications requiring extracellular recordings, the efficiency of the charge-delivery capacity of the electrodes material is of lower priority. However, low impedance and long-term stability of the interface is an important requirement and IrOx electrodes provide both of these requirements. This short paper will examine and discuss the use of arrays of activated IrOx electrodes, and their use for short-term in vitro extracellular recording of neuronal electrical activity. We report preliminary results employing neocortical acute tissue slices of the rat brain, selected as a target example to characterise $\mathrm{IrOx}$ electrode ageing and the recording of weak spontaneous electrical activity (Egert et al., 2002).In this context, in vitro simultaneous multisite recording of the spontaneous and evoked electrical activity of the nervous system represent an important step towards the understanding of the network-level and single-cell correlates of many (patho)physiological mechanisms (Rinaldi et al., 2007; Silberberg and Markram, 2007), as well as a crucial requirement for the validation of large-scale mathematical models and theories of the brain (Markram, 2006; Traub et al., 2005).

\section{MATERIALS AND METHODS IrOx MICROELECTRODES ARRAYS}

Microelectrode arrays (MEAs) were fabricated in clean rooms at the Institute of Microtechniqe of the EPFL and Institute of Electrical Engineering I at RWTH Aachen University, through a custom technological process (Figure 1). Briefly, a reversal photoresist lift-off 


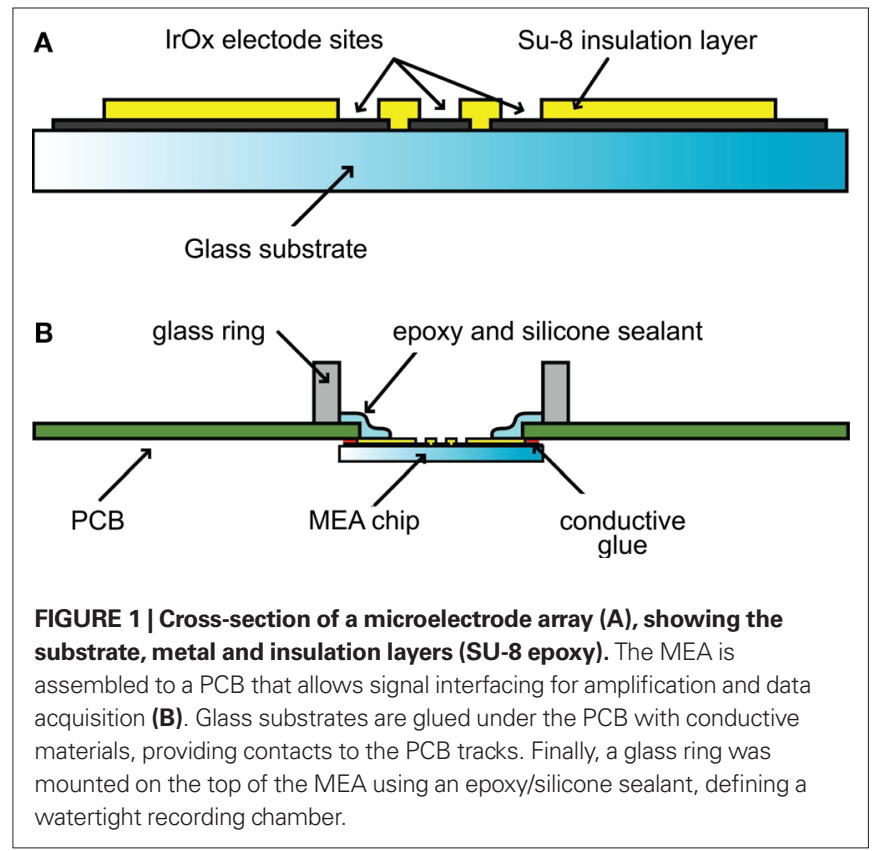

technique (AZ5214, Clariant, Germany) was employed to pattern metal using a glass substrate. The iridium layer was sputter-deposited as described in Wessling et al. (2007), with the following parameters: $180^{\circ} \mathrm{W}$ - DC Power, $100 \mathrm{sccm}$ - Argon flow and $10.4 \mathrm{sccm}$ - oxygen flow. A layer of photosensitive SU-8 $(5 \mu \mathrm{m})$ was finally used as an insulating layer (Lorenz et al., 1997), covering the iridium leads while leaving free access to the electrodes sites and contacts (Figure 1A).

The MEA layout consisted in a $12 \times 10$ square grid arrangement of 120 circular microelectrodes, each measuring $30 \mu \mathrm{m}$ in diameter and located $100 \mu \mathrm{m}$ apart from each other (Figure 2B). MEAs were glued onto printed circuit boards (PCB - Figures $1 \mathrm{~B}$ and 2) by a screen-printing technique, to provide external interfacing to multichannel amplifying electronics (Figure 2A). Platinum-based MEAs, with identical layout and electrode geometry, were also fabricated (Nam et al., 2006), and employed for comparison. Finally, the recording chamber was sealed with epoxy and silicone sealant (e.g. Sylgard 184, Distrelec, Switzerland) to ensure watertight connection of the MEA (Figure 1B).

\section{PREPARATION OF BRAIN SLICES AND PHARMACOLOGY}

Brain slices $(300 \mu \mathrm{m}$ thick) were prepared from Wistar rats, on postnatal days 15-20, by standard procedures (Köndgen et al., 2008), in accordance with institutional and national guidelines (Cantonal Veterinary Service, license n. 1550.1 to M.G.). Briefly, brains were quickly excised and rinsed in ice-cold $\left(<4^{\circ} \mathrm{C}\right)$ artificial cerebrospinal fluid (aCSF; containing in mM: $125 \mathrm{NaCl}, 25$ $\mathrm{NaHCO}_{3}, 2.5 \mathrm{KCl}, 1.25 \mathrm{NaH}_{2} \mathrm{PO}_{4}, 2 \mathrm{CaCl}_{2}, 1 \mathrm{MgCl}_{2}, 25$ glucose, continuously bubbled with $\left.95 \% \mathrm{O}_{2}, 5 \% \mathrm{CO}_{2}\right)$. Parasagittal neocortical slices were cut with a vibratome (VT1000S; Leica Microsystems, Wetzlar, Germany), trimmed down to an area of about $3 \times 2 \mathrm{~mm}^{2}$ centered to the primary somatosensory area (S1), and incubated at $35^{\circ} \mathrm{C}$ for $45 \mathrm{~min}$. Slices were afterward left at room temperature $\left(24^{\circ} \mathrm{C}\right)$. Electrophysiological recordings were carried out at room temperature, perfusing slices with an extracellular solution that differed from the aCSF in the concentration of potassium, calcium and magnesium ions (i.e. $6.25 \mathrm{KCl}, 1.5 \mathrm{CaCl}_{2}, 0.5 \mathrm{MgCl}_{2}$, bubbled with $95 \% \mathrm{O}_{2}, 5 \% \mathrm{CO}_{2}$ ). This results in an increase of the basal level of spontaneous electrical activity, as in Berger et al. (2006) (see also Richardson et al., 2005). In some experiments, NMDA ( $N$-methylD-aspartic acid, 10-100 $\mu \mathrm{M}$ ) was applied to mimic the non-selective activation of glutamatergic NMDA-receptors and further probe network-level excitability. All the chemicals were obtained from Sigma-Aldrich or Merck (Switzerland).

\section{SURFACE COATING OF THE MEAs}

MEAs were used repeatedly and cleaned before each experiment with methanol and distilled water. To improve mechanical adhesion of the tissue slice, the MEA surfaces was routinely coated with cellulose nitrate (CN, Schleicher \& Schuell, Dassel, Germany) dissolved in methanol $(0.14 \mathrm{mg} / \mathrm{ml}) .4-6 \mu \mathrm{l}$ of this solution was spread out around the electrode area and dried in air. A glass ring (diameter $20 \mathrm{~mm}$ ), glued to the PCB base plate with silicone rubber (Sylgard 184, World Precision Instruments, Berlin, Germany), formed a recording chamber with a volume of $1.5 \mathrm{ml}$.

\section{RESULTS}

\section{IRIDIUM ACTIVATION PROCESS}

IrOx microelectrodes were activated and restored to the activated state using a simple electrical protocol: each MEA electrode was connected to a potentiostat (AUTOLAB PGSTAT302, Echochemie, The Netherlands), with the counter and the reference terminals connected to a small platinum plate electrode, dipped into the phosphate buffer solution (PBS $10 \mathrm{mM}$ phosphate buffer, $2.7 \mathrm{mM} \mathrm{KCl}$ and $137 \mathrm{mM} \mathrm{NaCl}, \mathrm{pH} 7.4$ at $25^{\circ} \mathrm{C}$, Sigma-Aldrich, Germany) with a contact surface of a few square millimeters. The MEA electrodes were used as the working electrodes (Bockris et al., 2000). For the sake of simplicity, all 120 electrodes were activated simultaneously by connecting them in parallel rather than using a long and tedious but more controlled serial process. This approach, may however, lead to an inhomogeneous current density, so that the degree of activation would differ from one electrode to another.

In order to study the activation, as well as the effects of aging and reactivation, a number of linear voltage sweep cycles were performed until the peak current at the sweep maximum and minimum voltage no longer increased from one cycle to the next. Four or five cycles, from 1.4 to $-1.4 \mathrm{~V}$ were found necessary to achieve this condition and thence surface activation. A number of trials were required to achieve successful activation and reproducible results. After one or two cycles, micrometer sized bubbles could occasionally form on top of the electrodes as the relatively high voltage used caused electrolysis of water. Since these bubbles were found to partially mask or shield the electrodes from the electrolyte, a small plastic needle was used to establish a liquid flow on top of the electrode surface and detach the bubbles, allowing the electrode activation process to resume. In addition, because of the platinum counter/reference electrode properties, the measured sweep current was sometimes asymmetric. In order to prevent this effect a DC voltage offset (up to $0.2 \mathrm{~V}$ ) was used to compensate for activation currents that were too large in magnitude.

After (re)activation, the electrode arrays were rinsed carefully in PBS solution and the electrode impedance characterized using a precision impedance analyzer (4294A, Agilent, USA). 


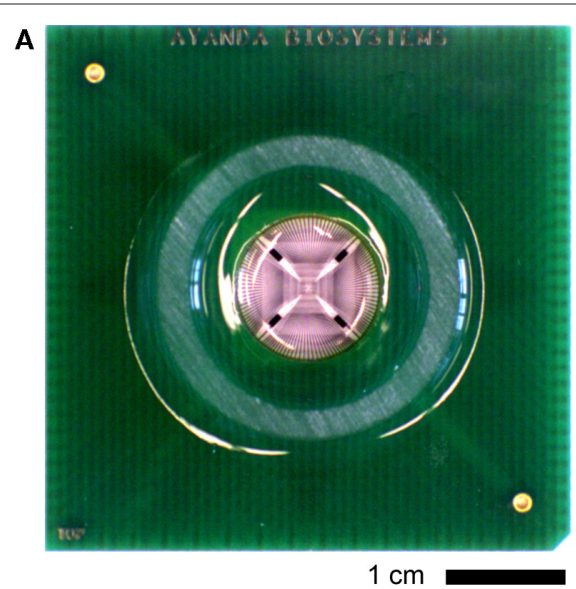

B

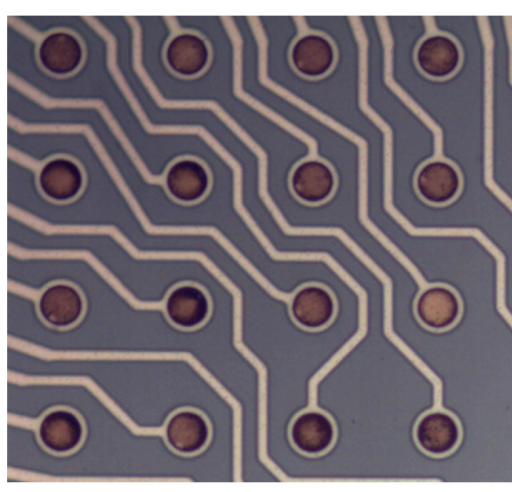

$100 \mu \mathrm{m}$
C

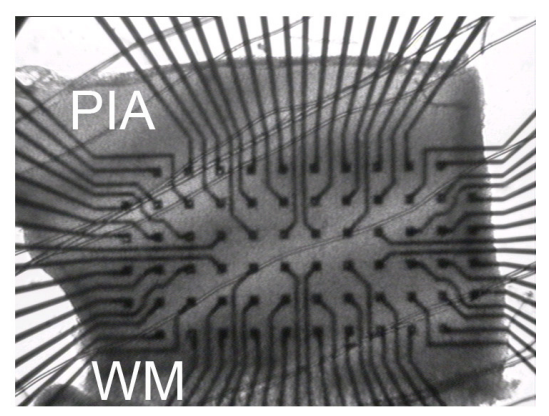

$0.6 \mathrm{~cm}$
FIGURE 2 |Top view of the full MEA assembled on a PCB (A) and close-up of the substrate electrode array (top-left corner) (B), showing 16 electrode sites and the insulated leads. Each microelectrode is $30 \mu \mathrm{m}$ in diameter, with a 100- $\mu \mathrm{m}$ spacing, arranged in a $12 \times 10$ square grid layout. A brain tissue slice (C), obtained acutely from the somatosensory rat cortex and comprising cellular layers between the pia and the white matter (WM), can be mechanically coupled to the MEA to record and stimulate the neuronal electrical activity (see Figure 6).

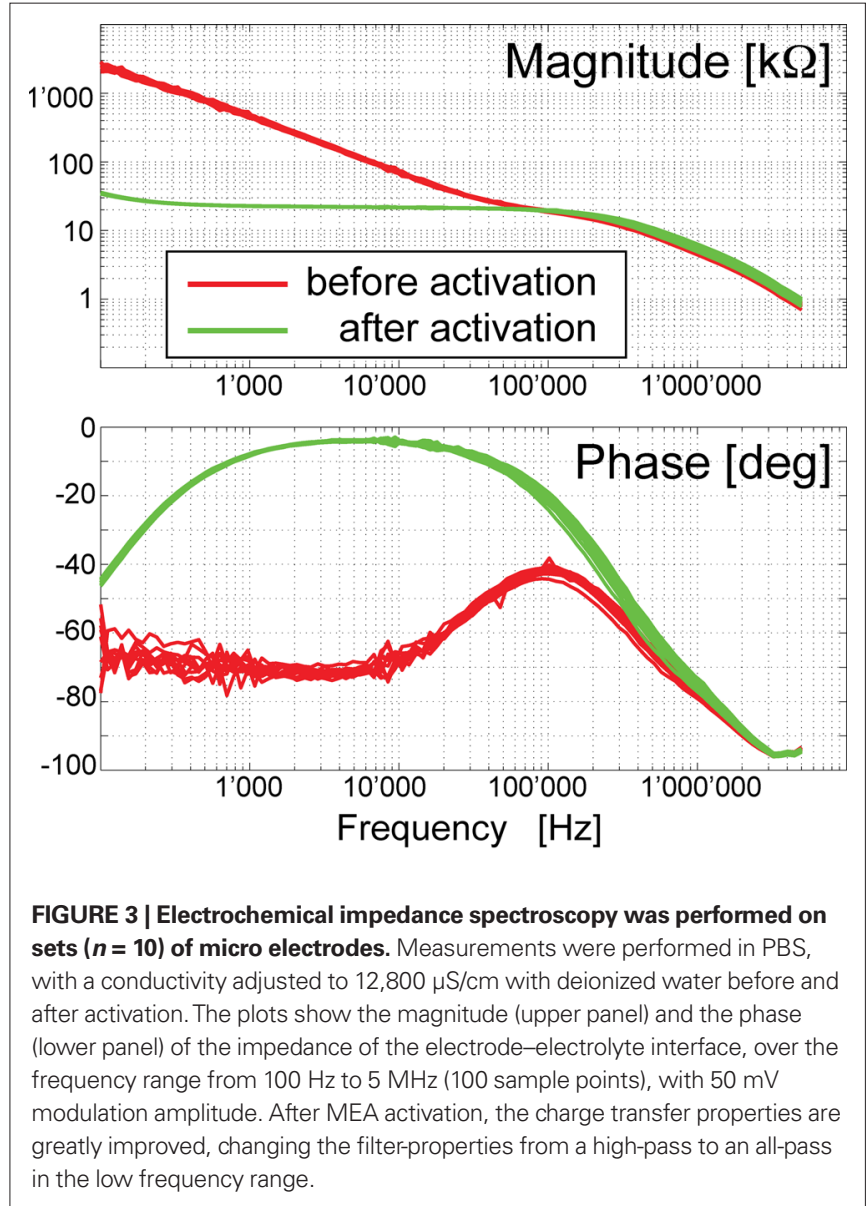

The results of the activation protocols are summarized in Figure 3, where the characteristic frequency of the electrode double layer impedance decreases by three orders of magnitude compared to inactivated electrodes (taken at $45^{\circ}$ phase point), from $10^{5}$ to

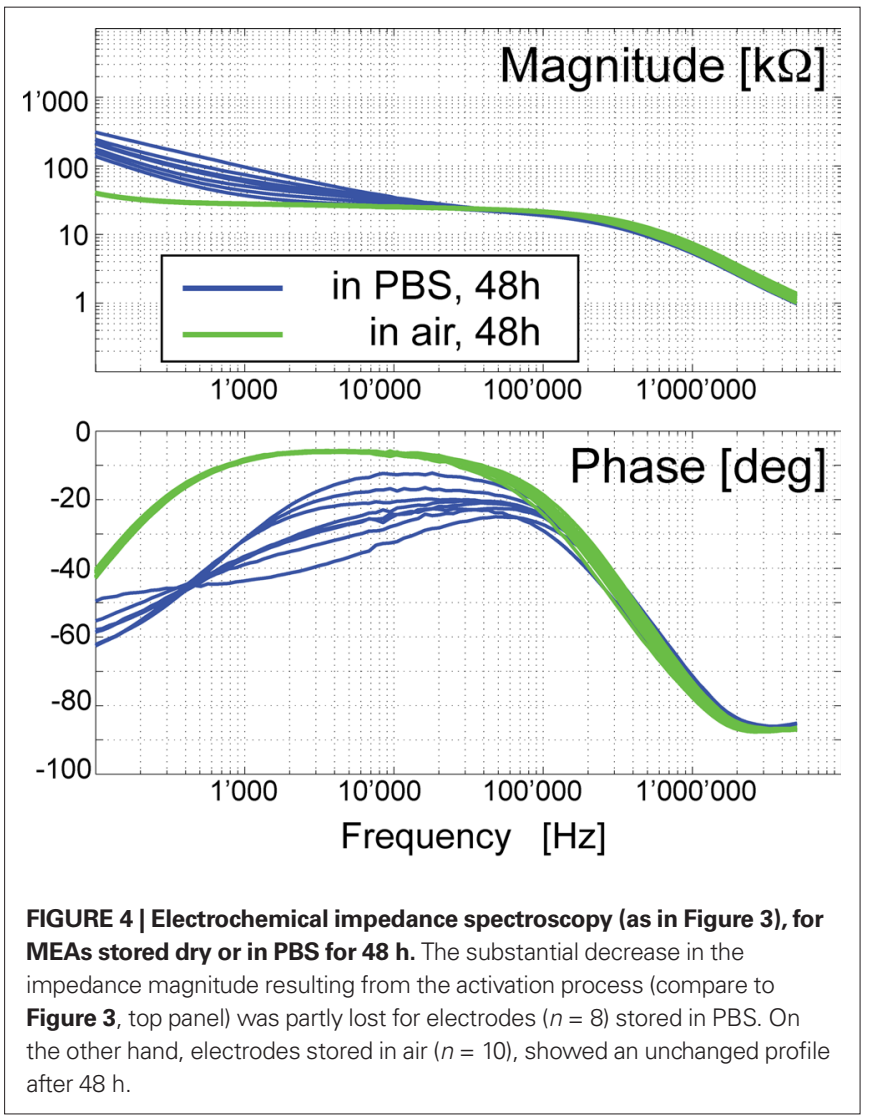

$10^{2} \mathrm{~Hz}$. The impedance homogeneity was also found to be very high, with differences only at higher frequencies probably resulting from stray capacitances in the leads rather than from heterogeneity in electrode surface properties.

Aging of the electrode impedance properties was determined by measuring the impedance after $48 \mathrm{~h}$, with electrodes stored in PBS or dry (Figure 4). Storing MEAs in a dry environment leads 
to a small increase in phase and impedance, by only a few percents. As expected, the electrode impedance reduced with time when MEAs were stored in PBS. After reactivation with one or two activation cycles, the original properties of the electrodes could be recovered.

\section{ELECTRODE IMAGING}

To further characterize the activation and aging effects of IrOx MEAs, topographic images were acquired using a digital holographic microscope capable of resolving vertical surface roughness in the $n m$ range (DHM R1000, Lyncée Tec SA, Switzerland). In this technique the in-plane resolution is still limited by diffraction, and was in the sub-micrometer range (Montfort et al., 2006).

As shown in Figure 5A-C, after digital reconstruction, the hologram of the electrode surface shows that the increase in roughness induced by activation is still present after 1 month of storage in dry conditions. For electrodes stored in saline, the surface had returned to an intermediate state, consistent with data in Figure 4. Figure 5D shows scanning electron microscopy images, indicating that the activated $\mathrm{IrOx}$ surface has nanometer scale roughness details.

\section{NOISE CHARACTERIZATION}

Noise analysis was performed on the raw voltage traces, recorded in sets of five, obtained with the electrolyte used in the electrophysiological experiments (i.e. aCSF - see Materials and Methods). Although the A/D 12-bits conversion resolution (i.e. $0.052 \mu \mathrm{V} /$ level) used for detecting neuronal activity was just able to resolve the very small signal-amplitude of the noise in IrOx electrodes, the distribution of the amplitudes was Gaussian, with a standard deviation of $0.66 \mu \mathrm{V}$ (for 181 planar IrOx electrodes). These excellent
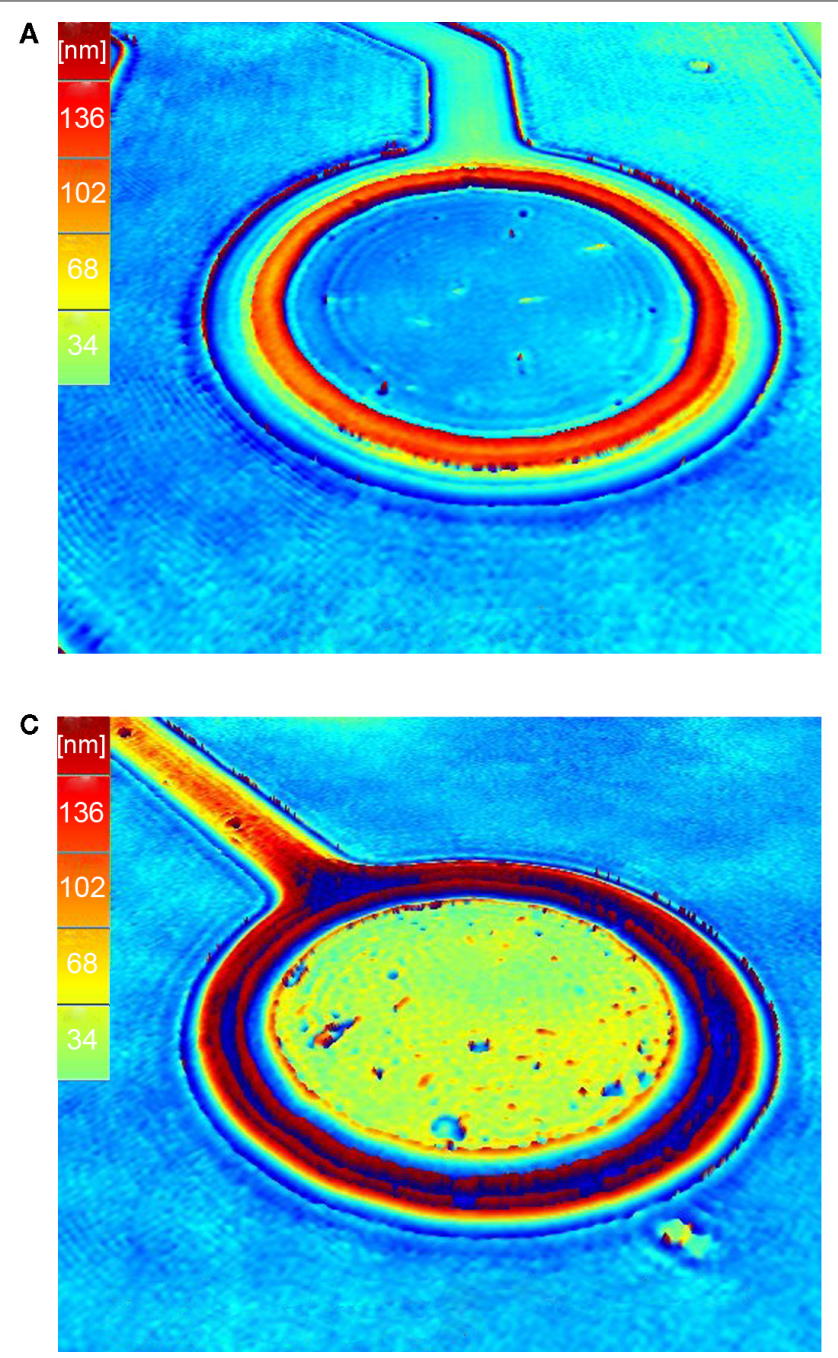

FIGURE 5 |The surface roughness of MEAs, before and after IrOx activation, studied by digital holographic microscopy (A-C) and scanning electron microscopy (D). The first technique reveals a substantial difference in the vertical nanometer scale surface roughness following IrOx activation (B), compared to electrode surface before activation (A). (B) Shows an
B

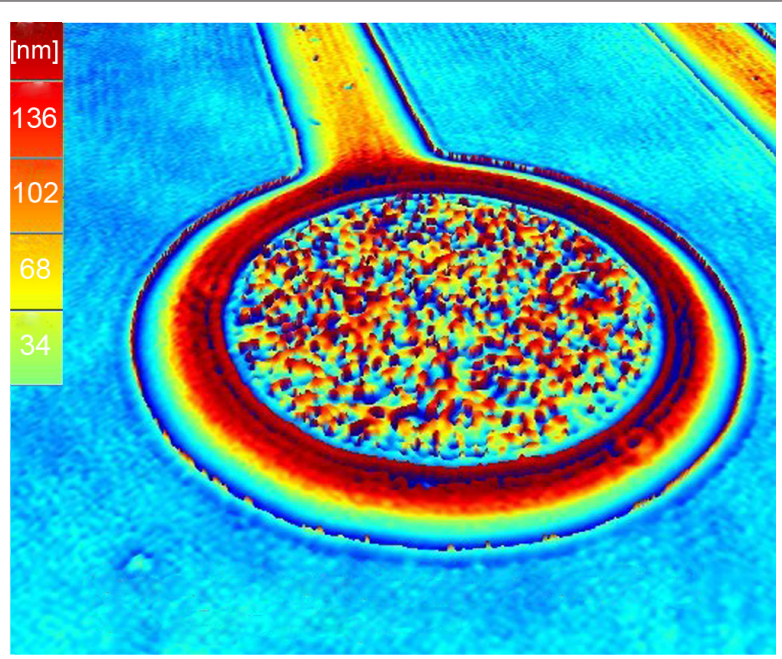

D

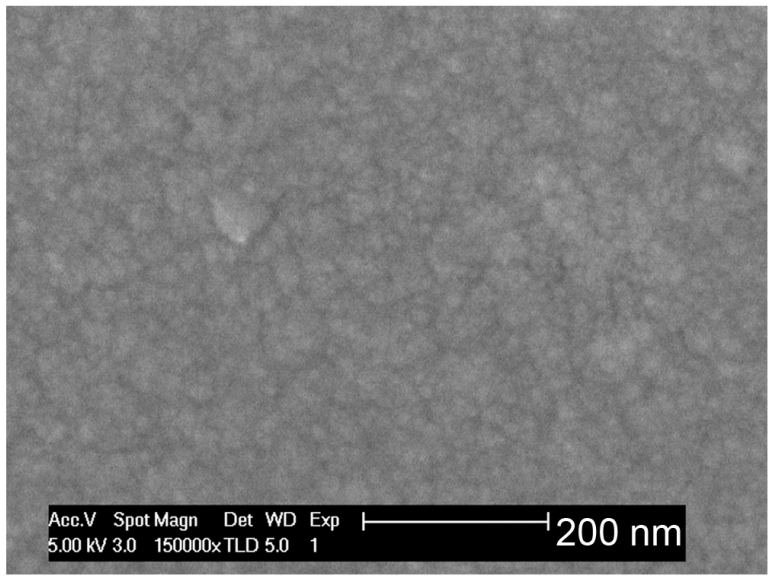

image of an electrode after 1 month of storage in air, whilst electrodes stored for 1 month in PBS (C) degenerate and return to a condition intermediate between (A) and (B). Scanning electron microscopy shows the nanometer scale surface roughness of an activated electrode (D). 
features can be compared to arrays of platinum microelectrodes, fabricated using an identical process and layout. The same noise analysis performed on these MEAs had 10-times worse performances (i.e. $5.7 \mu \mathrm{V}$, across 123 planar Pt electrodes).

\section{ELECTROPHYSIOLOGICAL RECORDINGS AND ANALYSIS}

Neuronal spiking activity was recorded extracellularly using the IrOx integrated thin-film MEAs as in (Egert et al., 2002). Slice alignment to the inner area of the MEA was performed manually under visual control, making each of the six cortical layers cover the electrodes.

After 5 min of accommodation time following the mounting of each slice on the MEAs, spiking activity was monitored and recorded for $30 \mathrm{~min}$. Signals from each electrode were amplified (1200 times) and band-pass filtered (300-3000 Hz) with a 120channels amplifier (Ayanda Biosystems SA, Lausanne, Switzerland). Raw signals were further sampled at $25 \mathrm{kHz} /$ channel from 120 channels simultaneously, and digitized (12-bit) through a 128-channel
PCI-bus A/D card (Multi Channel Systems, Reutlingen, Germany) for off-line analysis. MCRack (Multi Channel Systems, Reutlingen, Germany) was used to acquire and store the data (2 Gb/session). Data were analyzed using Matlab (The Mathworks, Natick, MA, USA) and custom software written in C. Raw data were digitally conditioned using second order Butterworth filters $150 \mathrm{~Hz}$ and $2.5 \mathrm{kHz}$ and fully rectified. The occurrence of an action potential was identified by a peak-detection algorithm, based on the crossing of an adaptive threshold (i.e. the LimAda algorithm of Wagenaar et al., 2006). Recorded events included the time-stamp of the spike, the index of the corresponding electrode, and the preceding $2 \mathrm{~ms}$ and following $4 \mathrm{~ms}$ of the corresponding voltage trace (Figure 6B). The conclusions reported here are based on repeated observations collected by MEAs on six cortical tissue slices.

Figure $\mathbf{6}$ is a sample of a recording, showing extracellular voltages recorded from a few IrOx electrodes. As discussed in the previous section, the good signal-to-noise ratio translates to a substantially higher probability of detecting single-unit activity

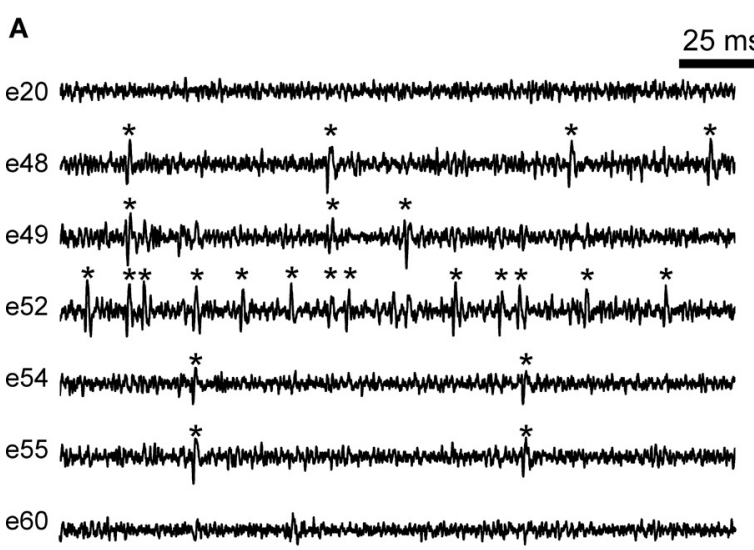

C

e2 e9

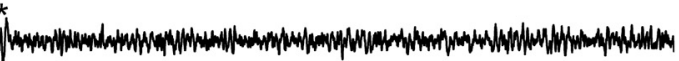

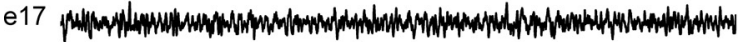

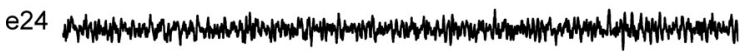

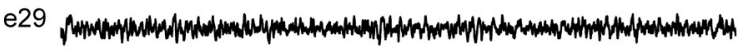

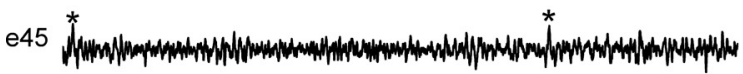

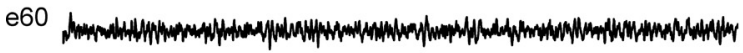

\section{B}
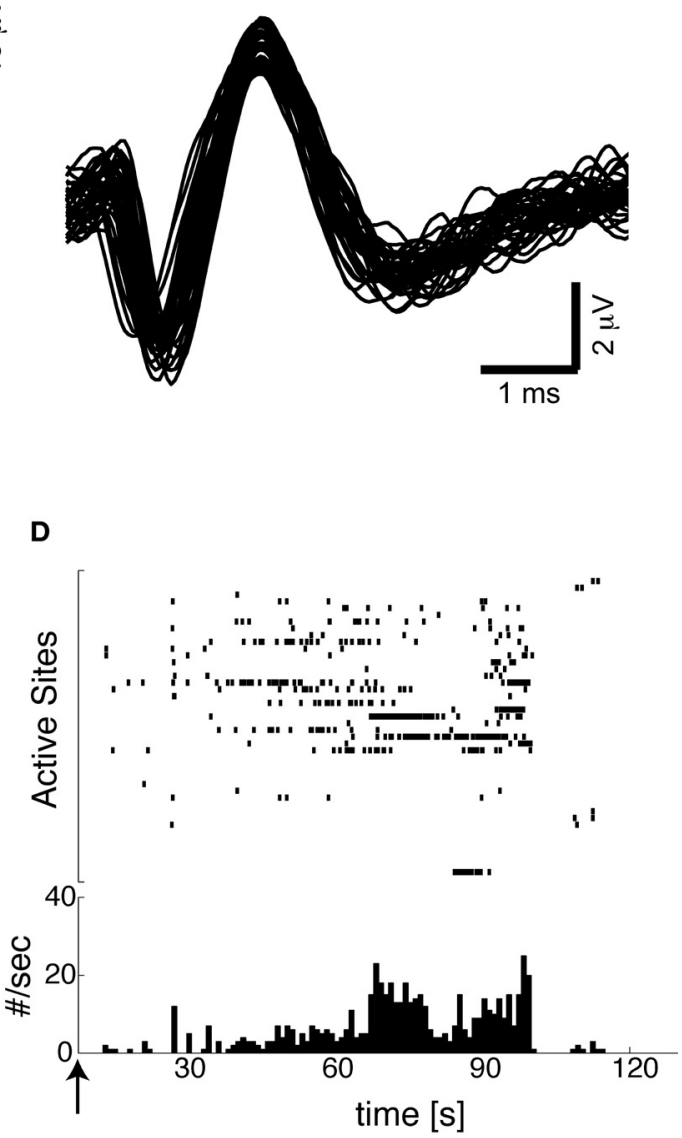

FIGURE 6 | Extracellular voltages, recorded from IrOx electrodes (A), demonstrates excellent signal-to-noise ratio in probing spontaneous spiking activity in acute slices of rat neocortex. Asterisks identify the occurrence of single-unit events, whose waveforms (B) resemble the secondderivative of an intracellular action potential, consistent with the expected behavior of the neuron-electrode electrochemical interface. (C) Due to a higher intrinsic noise, planar platinum MEAs allow only the detection of strong signals and generally transduce less neuronal activity. Transient collective network responsiveness to bath application of an agonist of glutamatergic excitatory synaptic receptors (i.e. NMDA ${ }_{\mathrm{R}}$ ), occurred at the time indicated by the arrow, and were simultaneously monitored by the IrOx MEAs electrodes. (D) Once detected by an adaptive-threshold peak-detection algorithm, spikes from individual electrodes are represented by the time of occurrence (top panel) or by the overall event rate, specified in bins of $1 \mathrm{~s}$ (bottom panel). 
in acute slices of rat neocortex. As discussed in Martinoia et al. (2004), single-unit events resemble higher time-derivative of an intracellular action potentials, consistent with the electrical properties reported in Figure 3. In preliminary results we found that $\mathrm{IrOx}$ MEAs detected more spontaneous activity than Pt-MEAs, although a more accurate quantitative comparison requires the same brain tissue slice to be removed from one MEA and mounted on another. Such a procedure was mechanically stressful for the tissue and it was not pursued in this study.

\section{DISCUSSION}

The extracellular spontaneous spiking activity level measured using the activated $\mathrm{IrOx}$ MEAs demonstrate that good results can be achieved with these planar electrodes. In general, for similar signal-to-noise ratios, 3D electrodes or platinum black electrodes have been proposed (Heuschkel et al., 2002), although at the cost of more complicated manufacturing process (3D devices) and less reproducible results (platinum black). The high reproducibility of the $\mathrm{IrOx}$ impedance data across the array (Figures 3 and 4 ) is due to the fact that the activation process self-limits because the current density saturates after a number of cycles. Electrodeposition of Pt-black is influenced by the distribution and the current density at the electrode surface, which varies from one electrode to another as well as during the deposition process through changes of electrode surface geometry. In addition, electrodeposition of Pt-black on 120 electrodes MEAs is particularly challenging and time consuming as it requires on-line monitoring of individual currents at each electrode throughout the entire deposition process.

The storage of the IrOx electrodes in dry conditions showed that electrode properties can be preserved. On the other hand, although a reactivation process is possible, electrode degradation is considerable in PBS.

Although the study degradation process would deserve more attention, it is likely that such an electrode aging is due to the $\mathrm{IrOx}$ returning to a lower oxidation level thus decreasing its effective surface, as its observed correlates are fully reversible. The electrical reactivation process is not compatible with the presence of biological samples, which makes the quality of long-term monitoring (over $48 \mathrm{~h}$ ) dependent on the electrode ageing.

Nevertheless, measurements over shorter time windows, particularly useful in slice electrophysiology, can be achieved with acceptable electrode properties. In such a case, the array can be then daily reactivated and cleaned a number of times, to restore the original electrode properties, reusing the same MEAs a number of times.

\section{ACKNOWLEDGEMENTS}

The authors are grateful to S. Garcia for excellent lab assistance and to L. Gambazzi for discussions. S.G. is grateful to E. Cuche for assistance with digital holographic microscopy and A. Tourvieille for assistance with scanning electron microscopy. M.G. and H.M. acknowledge support from the EPFL and the European Commission “NEURONANO” FP6 grant (NMP4-CT-2006-031847).

\section{REFERENCES}

Berger, T., Lüscher, H.-R., and Giugliano, M. (2006). Transient rhythmic network activity in the somatosensory cortex evoked by distributed input in vitro. Neuroscience 140, 1401-1413.

Blau, A., Ziegler, C., Heyer, M., Endres, F., Schwitzgebel, G., Matthies, T., Stieglitz, T., Meyer, J.U., and Gopel, W. (1997). Characterization and optimization of microelectrode arrays for in vivo nerve signal recording and stimulation. Biosens. Bioelectron. 12, 883-892.

Bockris, J.O'M., Reddy, A. K. N., and Gamboa-Aldeco, M. (2000). Modern Electrochemistry 2A: Fun damentals of Electrodics, 2nd Edn. New York, Kluwer Academic/Plenum Publishers.

Cogan, S. F. (2006). In vivo and in vitro differences in the charge-injection and electrochemical properties of iridium oxide electrodes. Conf. Proc. IEEE Eng. Med. Biol. Soc. 1, 882-885.

Cogan, S. F., Troyk, P. R., Ehrlich, J., and Plante, T. D. (2005). In vitro comparison of the charge-injection limits of activated iridium oxide (AIROF) and platinum-iridium microelectrodes. IEEE Trans. Biomed. Eng. 52, 1612-1614.
Egert, U., Heck, D., and Aertsen, A. (2002). Two-dimensional monitoring of spiking networks in acute brain slices. Exp. Brain Res. 142, 268-274.

Gabay, T., Ben-David, M., Kalifa, I., Sorkin, R., Abrams, Z., Ben-Jacob, E., and Hanein, Y. (2007). Electro-chemical and biological properties of carbon nanotube based multi-electrode arrays. Nanotechnology 18, 035201-035207.

Gabriel, G., Gómez-Martínez, R., and Villa, R. (2008). Single-walled carbon nanotubes deposited on surface electrodes to improve interface impedance. Physiol. Meas. 29, S203-S212.

Heuschkel,M.O.,Fejtl,M.,Raggenbass, M., Bertrand, D., and Renaud, P. (2002). A three-dimensional multi-electrode array for multi-site stimulation and recording in acute brain slices. J. Neurosci. Methods 114, 135-148.

Keefer, E. W., Botterman, B. R., Romero, M. I., Rossi, A. F., and Gross, G.W.(2008).Carbon nanotubes coating improves neuronal recordings. Nat. Nanotechol. 3, 434-439.

Köndgen, H., Geisler, C., Fusi, S., Wang, X. J., Lüscher, H. R., and Giugliano, M. (2008). The dynamical response properties of neocortical neurons to temporally modulated noisy inputs in vitro. Cereb. Cortex $18,2086-2097$.
Lorenz, H., Despont, M., Fahrni, N., LaBianca, N., Renaud, P., and Vettiger, $\mathrm{P}$. (1997).SU-8: a low-cost negative resist for MEMS. J. Micromech. Microeng. 7, 121-124.

Markram,H.(2006). The Blue Brain project. Nat. Rev. Neurosci. 7, 153-160.

Martinoia, S., Massobrio, P., Bove, M., and Massobrio, G. (2004). Cultured neurons coupled to microelectrode arrays: circuit models, simulations and experimental data. IEEE Trans. Biomed. Eng. 51, 859-864.

Marzouk, S. A., Ufer, S., Buck, R. P. Johnson, T. A., Dunlap, L. A., and Cascio, W.E. (1998). Electrodeposited iridium oxide $\mathrm{pH}$ electrode for measurement of extracellular myocardial acidosis during acute ischemia. Anal. Chem. 70, 5054-5061.

Mazzatenta, A., Giugliano, M. Campidelli, S., Gambazzi, L. Businaro, L., Markram, H., Prato, M., and Ballerini, L. (2007). Interfacing neurons with carbon nanotubes: electrical signal transfer and synaptic stimulation in cultured brain circuits. J. Neurosci. 27, 6931-6936.

Mokwa, W. (2007). Artificial retinas. In Comprehensive Microsystems, Vol. 3, Y. B. Gianchandani, O. Tabata and H. Zappe, eds (Oxford, Elsevier Science), pp. 201-217.
Montfort, F., Colomb, T., Charrière, F. Kühn, J., Marquet, P., Cuche, E., Herminjard, S., and Depeursinge, C. (2006). Submicrometer optical tomography by multiple-wavelength digital holographic microscopy. Appl. Opt. 45, 8209-8217.

Nam, Y., Wheeler, B. C., and Heuschkel, M. O. (2006). Neural recording and stimulation of dissociated hippocampal cultures using microfabricated three-dimensional tip electrode array. J. Neurosci. Methods 155, 296-299.

Richardson, M. J., Melamed, O., Silberberg, G., Gerstner, W., and Markram, H. (2005). Short-term synaptic plasticity orchestrates the response of pyramidal cells and interneurons to population bursts. $J$. Comp. Neurosci. 18, 323-331.

Rinaldi, T.,Silberberg, G., and Markram, H. (2007). Hyperconnectivity of local neocortical microcircuitry induced by prenatal exposure to valproic acid. Cereb. Cortex 18, 763-770.

Silberberg, G., and Markram, H. (2007). Disynaptic inhibition between neocortical pyramidal cells mediated by Martinotti cells. Neuron 53, 735-746.

Traub, R., Contreras, D., and Whittington, M. (2005). Combined 
experimental/simulation studies of cellular and network mechanisms of epileptogenesis in vitro and in vivo. J. Clin. Neurophysiol. 22, 330-342.

Wagenaar, D. A., Pine, J., and Potter, S. M. (2006). An extremely rich repertoire of bursting patterns during the development of cortical cultures. BMC Neurosci. 7, 11.

Wessling, B., Besmehn, A., Mokwa, W., and Schnakenberg, U. (2007). Reactively sputtered iridium oxide influence of plasma excitation and substrate temperature on morphology, composition, and electrochemical characteristics. J. Electrochem. Soc. 154, F83-F89.

Wessling, B., Mokwa, W., and Schnakenberg, U. (2006). RFsputtering of iridium oxide to be used as stimulation material in functional medical implants. J. Micromech. Microeng. 16, S142-S148.

Conflict of Interest Statement: The authors declare that the research was conducted in the absence of any commercial or financial relationships that could be construed as a potential conflict of interest.

Received: 06 August 2008; paper pending published: 11 September 2008; accepted: 15 January 2009; published online: 22 January 2009.

Citation: Gawad S, Giugliano M, Heuschkel M, Wessling B, Markram $H$, Schnakenberg $U$, Renaud $P$ and Morgan $H$ (2009) Substrate arrays of iridium oxide microelectrodes for in vitro neuronal interfacing. Front. Neuroeng. (2009) 2:1. doi 10.3389/neuro.16.001.2009

Copyright (c) 2009 Gawad, Giugliano, Heuschkel, Wessling, Markram, Schnakenberg, Renaud and Morgan. This is an open-access article subject to an exclusive license agreement between the authors and the Frontiers Research Foundation, which permits unrestricted use, distribution, and reproduction in any medium, provided the original authors and source are credited. 